\title{
Factores que influyen en la elección de las carreras de pedagogía en Colombia'
}

Elias Said-Hung"

Gloria Gratacós ${ }^{\text {III }}$

Jorge Valencia Cobos ${ }^{\mathrm{IV}}$

\section{Resumen}

El presente trabajo tiene como objetivo identificar los factores que influyen sobre las motivaciones, la satisfacción y expectativas en torno al ejercicio profesional en estudiantes de las facultades de Educación y ciclos complementarios de Escuelas Normales Superiores (ENS) en Colombia, tomando caso de estudio la región Caribe de este país. Para ello, se aplicó un estudio de tipo cuantitativo, cuya muestra estuvo integrada por 805 estudiantes de programas afines a los antes mencionados, quienes estuvieron a cargo del diligenciamiento de una encuesta orientada a la medición de los factores que influyen en la elección de estos estudios, de acuerdo con la escala FIT-choice (Factors Influencing Teaching choice), desarrollado por Watt y Richardson (2007). Los resultados obtenidos no solo nos permiten establecer un perfil socioeducativo de los futuros maestros analizados en este trabajo; sino también, facilitan la identificación de los factores vinculados a los principales tipos de motivaciones que tienen mayor peso, al momento de elegir carreras relacionadas con la Pedagogía; siendo las motivaciones de tipo altruistas e intrínsecas las más valoradas por los estudiantes encuestados. Las motivaciones extrínsecas, relacionadas con el salario y la conciliación, por ejemplo, tuvieron una valoración menor, con la excepción del factor relacionado con la seguridad del trabajo. La satisfacción de la elección es elevada pese al bajo salario, la falta de estatus social y una alta disuasión social.

I- Este artículo expone parte de los resultados del proyecto vinculado al contrato número
2014-0401, financiado por Departamento Motivación profesores - Elección carrera - FIT choice - Colombia. Administrativo de Ciencia, Tecnología e Innovación, Colciencias.

II- Universidad Internacional de la Rioja. Madrid, España.

Contacto: esaidh@gmail.com

III- Centro Universitario Villanueva. Madrid, España.

Contacto: ggratacos@villanueva.edu

IV- Universidad del Norte, Barranquilla, Colombia.

Contacto: javalenciac@uninorte.edu.co 


\title{
Factors affecting the choice of teaching programs in Colombia
}

\author{
Elias Said-Hung \\ Gloria Gratacós" \\ Jorge Valencia Cobos III
}

\begin{abstract}
This paper aims to identify the factors that influence motivation, satisfaction and expectations about the professional practices among students in education colleges and postsecondary education colleges in Colombia, studying the case of the Caribbean region, based on a quantitative study with 805 students linked with the programs mentioned above, who were in charge of measuring the factors that influence the selection of teaching careers, according to the FITchoice scale (Factors Influencing Teaching choice), developed by Watt and Richardson (2007). Findings not only allow us to establish a socio-educational profile of future teachers analyzed in this work, but also help us to identify factors linked to the main types of motivations that mostly affect the choice of education careers. In this case, the altruistic and intrinsic motivations are the most valued by respondents, compared with extrinsic motivations, related to salary and time for family, for example, whose valuation was lower. Satisfaction with choice is well valued despite low salaries, lack of social status and high social dissuasion.
\end{abstract}

\section{Keywords}

Teacher motivation - Career choice - FIT choice - Colombia.

I- This article presents an overview

of the project, sponsored by Administrative Department of Science, Technology and Innovation, Colciencias (contract number 2014-0401).

II- Universidad Internacional de la Rioja. Madrid, Spain.

Contact: esaidh@gmail.com

III- Centro Universitario Villanueva.

Madrid, Spain.

Contact: ggratacos@villanueva.edu

IV- Universidad del Norte, Barranquilla, Colombia.

Contact: javalenciac@uninorte.edu.co 


\section{Introducción}

Es incuestionable el papel que juega la educación en el desarrollo económico de un país, donde la actual crisis mundial ha evidenciado, de nuevo, la importancia del desarrollo del capital humano. Priorizar el tema de la calidad de la educación requiere reconocer el papel de los profesores como la pieza clave de la mejora educativa (DELORS, 1996; BARBER; MOURSHED, 2007; DE MOURA; IOSCHPE, 2007). La OREALC/ UNESCO (2012) destaca la idea de diseñar e implementar carreras destinadas a fortalecer la profesión docente e incidir en la atracción de buenos candidatos. En el 2013 la Unión Europea publicó un estudio en 34 países para evaluar el atractivo de la profesión docente y las políticas que se estaban realizando para mejorarla. En este estudio se destaca la importancia de establecer medidas que ayuden a atraer, seleccionar y retener a los mejores para avanzar en el incremento de la educación, desde la diversidad de situaciones de enseñanza y competencias requeridas para afrontar la diversidad de situaciones que deben ser tenidas en cuenta por los docentes a cargo de la formación de los estudiantes (CARLO et al., 2013).

En América Latina, como bien destacan Vaillant (2013) y Cox, Beca y Cerri (2014), uno de los aspectos que ha centrado el debate, en el interior del sector educativo y en la agenda política regional, es el rol que tienen los docentes en la búsqueda de una mayor calidad y equidad. A pesar de lo antes expuesto, en la práctica aún está presente la paradoja de la falta de relevancia que tienen los docentes en el diseño de las políticas educativas a nivel regional (BECA; CERRI, 2014). Esto ha traído consigo una creciente puesta de atención en el aumento de las condiciones salariales e incentivos, desatendiendo otros aspectos igualmente importantes, como es la formación brindada a los futuros docentes y la búsqueda de mecanismos orientados a atraer los estudiantes mejor calificados para el ejercicio de esta importante profesión, a nivel social. Como bien apuntan Gatti, Siqueira y Dalmazo (2011), se han llevado a cabo, desde los diferentes sistemas educativos latinoamericanos, acciones abocadas a favorecer el acceso a plazas de docentes, sin centrarse también en la calidad de los currículos formativos brindados a estos nuevos profesionales. Algo que ha conllevado un marco de críticas orientadas a señalar la organización burocrática, la falta de articulación entre la teoría y la práctica recibida por los futuros docentes, así como la poca vinculación con la escuela, durante este proceso formativo (VAILLANT; MARCELO, 2012).

Estudios como los realizados por la UNESCO (2012) han dejado en claro el actual proceso de transición que viven muchos países de América Latina, en cuanto a la heterogeneidad del perfil de los docentes y del creciente cambio generacional para la reposición de los miembros de este colectivo, a favor de lograr la cobertura universitaria a nivel de secundaria y la mejora de los servicios educativos brindados a nivel de educación primaria. Un contexto que ha estado marcado, de acuerdo con Cox, Beca y Cerri (2014), por factores tales como: un marco heterogéneo ${ }^{1}$ de formación dirigida a los estudiantes interesados en convertirse en docentes; la variación en los estudios que oscila entre los 2 y 5 años, de acuerdo con el nivel de educación donde se insertara el futuro profesor (por ejemplo, en Colombia, la formación de profesores de primaria dura en torno a tres años, mientras que a nivel secundaria puede durar más tiempo); y la coexistencia aún de dos modelos de formación de los futuros docentes (los que adquieren una enseñanza disciplinar $\mathrm{y}$ pedagógica, y los que se convierten en licenciados de diferentes disciplinas ejercidas a nivel escolar, sin contar con una formación pedagógica conveniente). Lo expuesto aquí nos lleva a mostrar un contexto regional que da cuenta del impacto que ha traído consigo

1- En el caso latinoamericano, dependiendo del nivel de enseñanza donde se esté formando el futuro docente, puede encontrarse con varios tipos de instituciones abocadas a tal fin. Ejemplo de ello tenemos a Brasil y Chile donde la formación está mayoritariamente a nivel universitario, mientras que en el caso colombiano y mexicano, la formación de docentes en educación inicial se centra en muchos casos en escuelas normales de nivel superior. 
los diferentes niveles de desarrollo que ha caracterizado el movimiento orientado a la profesionalización del rol docente, el cual no ha estado exento del impacto de actores internos (por ejemplo gremios, así como los docentes ya en ejercicio), como externos (Ministerios de Educación, sector políticos y sector empresarial), quienes ejercen presión en el desarrollo de temas educativos, a nivel de cada país (SCHWARTZMAN, 2011; SCHMELKES, 2014), pero sobre todo han venido incidiendo en la forma cómo se ha desarrollado el marco heterogéneo antes descrito.

América Latina se encuentra ante dos grandes desafíos: por un lado, aumentar la oferta de docentes, cada vez mejor preparados para cada nivel del sistema educativo; y por otro, mejorar las ofertas educativas orientadas a la formación de profesionales dentro de este sector, con el fin de atraer el mayor número de candidatos calificados para el ejercicio de esta importante función social (CEPAL; UNESCO, 1996; VAILLANT, 2004; BARRERA-OSORIO; MALDONAD0; RODRIGUEZ, 2012; OREALC/ UNESCO, 2012). Es en la capacidad de asumir estos retos que cada país de la región contará con la suficiente capacidad para afrontar una mejora en el desempeño de los alumnos y de la calidad de la educación, en general. Un contexto que, como bien señalan García y otros autores (2014), al menos en el caso colombiano, el análisis de las diferencias en los resultados obtenidos en las pruebas PISA de 2011, entre las escuelas con excepcional y pobre desempeño, permiten dar cuenta de la importancia que tienen los docentes en los resultados de aprendizaje demostrados por los estudiantes en el marco de dichas pruebas, por encima de otras dimensiones (por ejemplo, la evaluación y el rendimiento de cuentas, la autonomía del centro o el liderazgo del rector). Algo que guarda relación con lo expuesto por autores como Rivkin, Hanushek y Kain (2005) o Hanushek (2011), en estudios realizados en los Estados Unidos, Canadá o Finlandia. Por lo que tener un buen o mal profesor puede suponer la diferencia del crecimiento en aprendizaje de un año entero (HUNT, 2009), si bien no hay un consenso sobre cuáles son las diferencias relevantes entre un buen y un mal profesor (BARRERA-OSORIO; MALDONADO; RODRIGUEZ, 2012).

\section{Factores que influyen en la elección de la carrera docente}

El establecimiento de políticas eficaces destinadas a fortalecer la profesión docente e incidir en la atracción de buenos candidatos, requiere partir de la identificación y comprensión de los factores que puedan atraer o disuadir a los candidatos en la elección de estudios de Pedagogía (DESHANO, 2012).

Pese a los esfuerzos en materia de políticas educativas en América Latina, la docencia no parece atraer aún a los mejores candidatos para el ejercicio de esta importante profesión. De hecho, los que ingresan a esta carrera formativa suelen tener un historial educativo promedio inferior a los que acceden a estudios más valorados socialmente (HUNT, 2009; BARON; BONILLA, 2011; VAILLANT, 2004; 2013).

Estudios realizados por Calvo (2006) y Vaillant (2013), destacan que la profesión docente parece estar siendo elegida por descarte; es decir, por no tener nota adecuada para entrar en otras opciones o como vía de acceso a otras opciones por los estudiantes con mejores resultados. Estos aspectos pueden explicar: 1) una alta tasa de deserción -que, en el caso de Colombia, supera el promedio nacional observado en dicho país (50,5\%, 2 puntos porcentuales por encima del resto)-; 2) el desinterés en los estudios de Pedagogía -según Gatti y otros autores (2008) solo un 2\% de los 1504 estudiantes entrevistados manifestaba querer estudiar para ser maestro y se da un muy bajo porcentaje de graduados en programas de Ciencias de la Educación (10\%), entre 2001 y 2013 , con respecto al resto de las áreas del conocimiento (COLOMBIA, 2016)-; 3) 
niveles de resultados muy pobres en la Prueba Saber $\mathrm{Pro}^{2}$, si se compara con el resto de áreas del conocimiento (ICFES, 2011).

En América Latina las tasas de abandono de la carrera docente en los primeros años de servicio no son tan altas como en países más desarrollados de la Organización para la Cooperación y el Desarrollo Económicos (OCDE), debido a que no existen mejores oportunidades laborales alternativas (ÁVALOS et al., 2010). No obstante, como destaca Ávalos (2014), en Chile se constatan tasas de abandono de alrededor del 40\%. Hecho que afecta, normalmente, a los mejores docentes, al tener más oportunidades de optar por puestos mejores en otras áreas profesionales que puedan mejores condiciones laborales y reconocimiento social (VAILLANT, 2010).

Autores como Tenti (2007) o Bellei y Valenzuela (2010), destacan la auto-percepción de los docentes en la región, como profesionales ubicados socialmente de clase media o media baja, con bajas remuneraciones, patrones de consumo precarios, trabajo con una alta carga de estrés y con poco tiempo para la planeación de sus labores diarias. Si bien las fuentes de satisfacción de los docentes son factores relacionados con su trabajo y la relación con sus colegas, y según Valle (2006), dos tercios de los profesores no dejarían la docencia por estar satisfechos, es generalizada, como hemos expuesto antes, la insatisfacción respecto a la subvaloración que la sociedad da a su profesión (ÁVALOS et al., 2010; GARCÍA et al., 2014). El escaso estatus de la profesión docente comparada con otras carreras universitarias da lugar en algunos casos a que los candidatos a ser maestros sean disuadidos por la presión social y por sus familiares a favor de otras alternativas con mejor prestigio social y mejor remuneradas (UNESCO, 2012).

En cuanto a las condiciones de trabajo, en la mayoría de los países la profesión docente goza de relativa estabilidad (FALUS;

\footnotetext{
2- Son las pruebas aplicadas a nivel de Colombia para comprobar el desarrollo de competencias adquiridas por los estudiantes durante su formación profesional.
}

GOLDBERG, 2011), aunque a nivel de los colegios privados esta condición es menor (tal es el caso de Colombia, Perú, Ecuador y Costa Rica). En lo que respecta a los sueldos, lo expuesto por Feldfeber (2007) sigue estando vigente, es decir siguen siendo bajos con respecto a lo que cobran egresados de otras titulaciones, a pesar que autores como Liang (1999) han dado cuenta de un contexto donde las diferencias salariales observadas entre docentes y otros profesionales no pareciera resultar tan elevadas. Algo que no termina de dejar de ser un fuerte foco de debate, tanto a nivel académico como social, ya que, esto último estaría en consonancia con los argumentos oficiales expuestos, por ejemplo, por el Ministerio de Educación Nacional de Colombia (NUMA, 2015), donde la diferencia salarial entre docentes y profesionales de áreas afınes a la salud e ingenierías, por ejemplo, se ubica en torno al 7\%, a favor de los profesionales ajenos al sector educativo, pese a que estudios como el de García y otros autores (2014) afirman lo contrario, es decir: que los profesores en instituciones públicas devengan un salario un 18\% inferior al que devengan los profesionales de la Medicina, Ingeniería, Derecho y Economía.

La solución a la baja calidad en la docencia no pasa únicamente por remuneraciones más altas, sino por el abordaje de otros factores que inciden en ello. Tal es el caso del fortalecimiento de los procesos formativos y apoyos pedagógicos dispuestos para el ejercicio de la labor docente a cargo (DE MOURA; IOSCHPE, 2007; KRISHNARATNE; WHITE; CARPENTER, 2013).

El estatus social alrededor del ejercicio docente no siempre está vinculado con salarios justos. En Alemania y Suiza los salarios son relativamente altos y sin embargo el respeto al docente es bajo (VARKEY GEMS FOUNDATION, 2013). Es por lo aquí expuesto que lo indicado, por ejemplo, por la UNESCO (2015), en cuanto a la necesidad de mejorar la consideración social de los profesores, resulta clave para asegurar una mayor motivación y satisfacción de estos. 
Todo ello, en favor de un mayor impacto en la retención de los docentes así como en el rendimiento de los estudiantes a su cargo.

Brookhart y Freeman (1992), así como Watt y Richardson (2007), identifican tres tipos de motivaciones, al momento de elegir los estudiantes carreras docentes: 1) motivaciones de tipo extrínsecas (inherentes al trabajo mismo como el salario, vacaciones, entre otras); 2) motivaciones de tipo intrínsecas (debido a aspectos propios de la profesión como el gusto por enseñar la materia); y 3) motivaciones de tipo altruistas (contribución de la profesión a la mejora de la sociedad). La clasificación propuesta por dichos autores, será tenida en cuenta en el presente trabajo, ya que permiten tener una aproximación más asertiva del tema propuesto, al menos a nivel de Colombia, para mejorar el atractivo y capacidad de retención de los buenos profesionales en la profesión.

Una idea generalizada en el debate alrededor de las motivaciones para el ejercicio de la carrera docente, es su relación con el nivel de compromiso que pueden tener los estudiantes, alrededor del desarrollo de su futura labor docente. De acuerdo con Ryan y Deci (2000) los niveles de compromiso suelen estar asociados más a razones intrínsecas que extrínsecas, es decir, motivaciones relacionadas con aspectos propios de la profesión, como por ejemplo el gusto por enseñar la materia. Así mismo, autores como Gratacós (2014), han indicado que los motivos por los que los alumnos eligen estudios en Educación son muy similares entre sí, sin importar los contextos socio-culturales o geográficos donde se ubiquen: motivos de tipo intrínsecos y motivos de tipo altruistas, por encima de motivaciones de tipo extrínsecas.

A nivel latinoamericano, estudios realizados por autores como Avendaño y González (2012) y García y otros (2014), en el caso de Chile y Colombia respectivamente, darían una base de confirmación de los expuestos por Ryan y Deci (2000) y Gratacós (2014). Ello en vista que las principales motivaciones expuestas por docentes o futuros profesores (estudiantes que se encontraban formándose en programas afines al área educativa), analizados por los autores antes mencionados, dan cuenta de aspectos intrínsecos y altruistas, relacionados con: el gusto por enseñar a niños y jóvenes, la percepción que tienen de la educación para la sociedad y el gusto por la asignatura impartida, entre otros aspectos.

Un rasgo que ha marcado hasta la fecha las investigaciones sobre aspectos motivacionales, al interior del sector educativo, ha sido la falta de consistencia metodológica, así como la ausencia de marcos de consenso teórico alrededor de este tema. Sobre todo cuando se intenta establecer un marco que ayude a categorizar los diferentes motivos en categorías superiores bien definidas (LÓPEZ-JURADO; GRATACOS, 2013). Esto supone la dificultad de estudiarlos a fondo para conocerlos mejor y por tanto, poder hacer planes de acción que ayuden a mejorar el atractivo de la profesión docente y acertar en los programas de formación de los futuros profesores. En especial cuando corresponde delinear políticas públicas que ayuden a una mayor atracción de mejores docentes. Algo que requiere un exhaustivo conocimiento de los factores que inciden en la decisión de ser maestro, a partir de la puesta en marcha de enfoques metodológicos que permitan la estandarización de mediciones y la realización de comparaciones de diferentes dimensiones y ámbitos (local, nacional e internacional).

Pese a la diversidad metodológica y conceptual tenida en cuenta hasta la fecha, estudios como los de Klassen y otros autores (2011) y Watt y otros autores (2012) han propuesto métodos consensuados que permiten hacer comparaciones entre los distintos países y así poder aprender de los efectos de las medidas tomadas. Tal es el caso del modelo de medición de la motivación, aplicado a la elección de la carrera docente, desarrollado por Watt y Richardson (2007), conocido como FIT-choice scale (escala de los factores que influyen en la elección de la carrera docente). Modelo que estimamos de utilidad para el abordaje del tema propuesto, al ofrecer una visión más completa 
del porqué las personas escogen la docencia como profesión (DESHANO, 2012) y de los factores presentes en estudios de motivación para ser profesor (BERGER; D’ASCOLI, 2012).

\section{Metodología}

Este estudio pretende identificar los factores que influyen sobre las motivaciones, la satisfacción y expectativas en torno al ejercicio profesional en estudiantes de las facultades de Educación y ciclos complementarios de Escuelas Normales Superiores (ENS) en Colombia, tomando caso de estudio la región Caribe de este país. Para ello, se tomó como instrumento de medición de los factores que influyen en la elección de estos estudios, la escala FIT-choice (Factors Influencing Teaching choice) desarrollado por Watt y Richardson (2007) y aplicado por primera vez en Australia. Posteriormente, ha sido aplicado en otros países: en Holanda (FOKKENS-BRUINSMA; CANRINUS, 2012), en Australia, USA, Alemania, Noruega (WATT et al., 2012), en Turquía (EREN; TEZEL, 2010; TOPKAYA; UZTOSUN, 2012), en Canadá y Omán (KLASSEN et al., 2011), en Suiza (BERGER; D'ASCOLI, 2012), en Alemania (FOKKENS-BRUINSMA; CANRINUS, 2012; KÖNIG; ROTHLAND, 2012), en Croacia (JUGOVI et al., 2013), China (LIN et al., 2012) y España (GRATACÓS, 2014).

Un aspecto importante a destacar de la elección de este instrumento es su aplicación en diferentes países con lo que nos ofrece la posibilidad de establecer comparaciones internacionales. Lo que nos permite ver cómo influyen las distintas políticas educativas aplicadas a la hora de hacer atractiva la profesión docente, teniendo siempre en cuenta las características propias de cada país que supone una clara adaptación al contexto de cada uno. Nos permite poder aprovechar las experiencias de otros contextos para generar conocimiento valioso y necesario para el desarrollo de un modelo de fortalecimiento de la docencia en la región caribe colombiana.

La encuesta aplicada fue la versión española del FIT choice (tabla 1) con el fin de medir los factores que influyen en la elección de la profesión docente, así como de las percepciones sobre ésta, desde la teoría de la expectativa del valor (expectancy-value theory), desarrollada por estudios como el de Eccles y otros autores (1993), que intenta explicar los determinantes de la motivación para la elección de carrera a través de las expectativas de éxito y la evaluación de la tarea. La escala FIT-choice desglosa los determinantes de la motivación en cuatro elementos: autopercepción, valor de la tarea, carrera de segunda opción y expectativas y creencias sobre la profesión. La autopercepción se refiere a la habilidad percibida para enseñar. El valor de la tarea comprende el valor intrínseco, valor de utilidad personal (seguridad del trabajo, movilidad laboral, conciliación familiar) y valor de utilidad social (influir en el futuro de los niños, mejorar la equidad social, hacer una contribución social y trabajar con niños/ jóvenes). Las expectativas y creencias de la profesión se explican con la exigencia de la profesión (carrera exigente y profesión exigente) y el retorno de la profesión (estatus social y salario). Por último, en carrera de segunda opción se tiene en cuenta el factor de si la carrera docente fue elegida por descarte. Por último, se incorporan aspectos de socialización anteriores a la elección (influencias de la familia, amigos y profesores) y percepciones de experiencias previas.

La encuesta recoge una primera parte de datos sociodemográficos y los factores motivacionales y de percepción (propiamente los del FIT choice) que son una escala Likert de 1 al 7, siendo 1 Nada de acuerdo y 7 Muy de acuerdo. Para su aplicación en Colombia se ha usado la adaptación del instrumento al español (GRATACÓS; LÓPEZ-JURADO, 2016). 


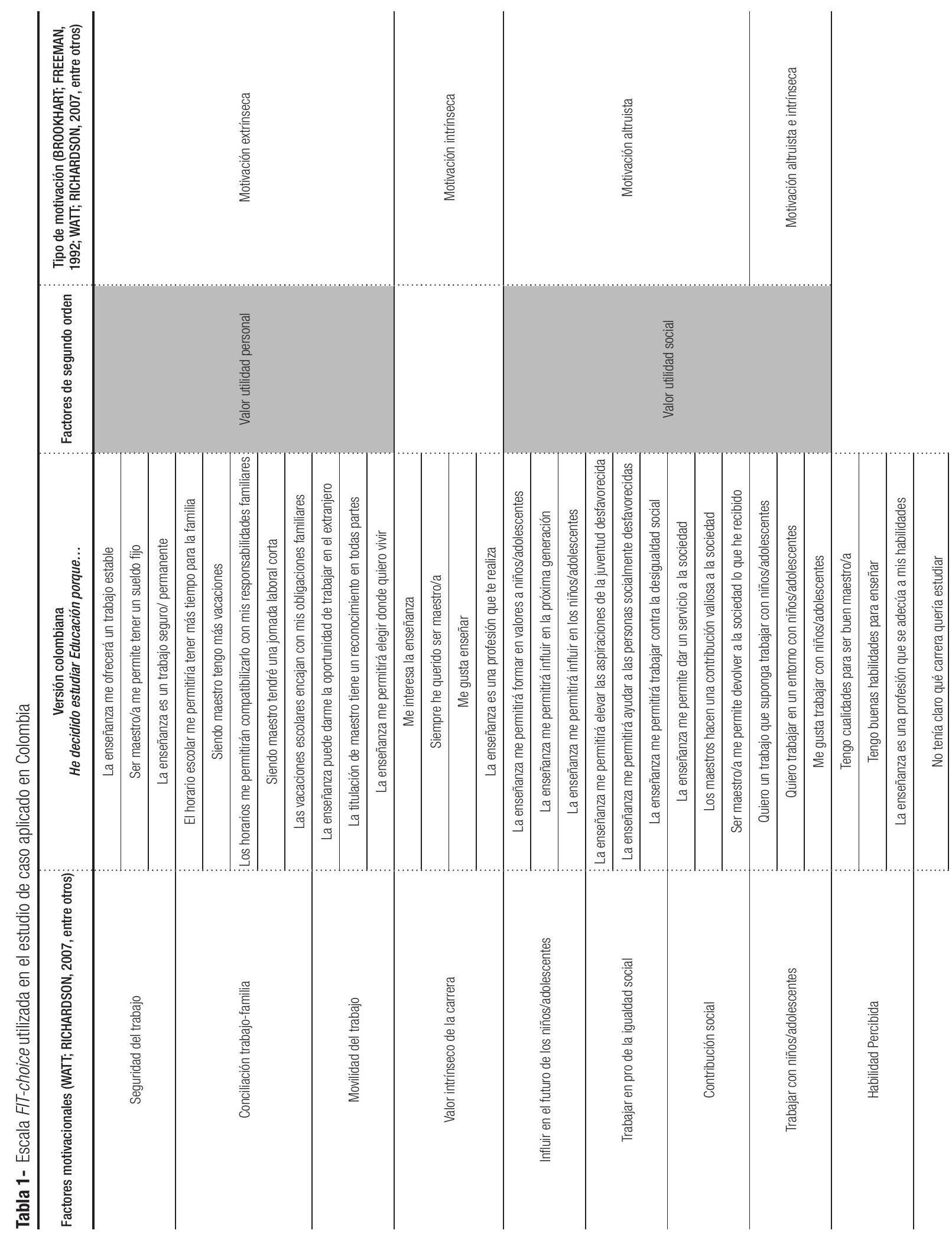


El presente trabajo parte de un estudio instituciones de educación superior (oficiales de tipo cuantitativo, cuya población de estudio y privada), los cuales ofertan diez programas (estudiantes vinculados a programas de relacionados con el área de pedagogía y en 21 pedagogía) se encontraban localizadas en siete ENS (oficiales y privadas) reflejado en la tabla 2.

Tabla 2 - Instituciones y programas de educación considerados para estudio

\begin{tabular}{|c|c|c|}
\hline Departamentos en Colombia & Institución & Programa \\
\hline \multirow{9}{*}{ Atlántico } & Corporación Universitaria Reformada & Licenciatura en Educación Bilingüe- Español e Inglés \\
\hline & \multirow[t]{2}{*}{ Universidad del Atlántico } & Licenciatura en Español y Literatura \\
\hline & & Licenciatura en Matemáticas \\
\hline & Escuela Normal Superior La Hacienda & Pedagogía \\
\hline & Escuela Normal Superior del Distrito de Barranquilla & Ciclos pedagógicos complementarios (programa general) \\
\hline & $\begin{array}{l}\text { Institución Educativa Escuela Normal Superior Nuestra Señora De } \\
\text { Fátima }\end{array}$ & Ciclos pedagógicos complementarios (programa general) \\
\hline & Institución Educativa Escuela Normal Superior Santa Ana de Baranoa & Ciclos pedagógicos complementarios (programa general) \\
\hline & Institución Educativa Escuela Normal Superior Santa Teresita & Ciclos pedagógicos complementarios (programa general) \\
\hline & Institución Educativa Normal Superior de Manatí & Ciclos pedagógicos complementarios (programa general) \\
\hline \multirow{3}{*}{ Bolívar } & Escuela Normal Superior de Cartagena de Indias & Ciclos pedagógicos complementarios (programa general) \\
\hline & Institución Educativa Normal Superior Montes de María & Ciclos pedagógicos complementarios (programa general) \\
\hline & Institución Educativa Normal Superior de Mompox & Ciclos pedagógicos complementarios (programa general) \\
\hline \multirow{4}{*}{ Cesar } & Universidad Popular del Cesar & $\begin{array}{l}\text { Licenciatura en Lengua Castellana e Inglés, Licenciatura } \\
\text { en Matemáticas y Física }\end{array}$ \\
\hline & & Licenciatura en Matemáticas y Física \\
\hline & Institución Educativa Normal Superior María Inmaculada & Ciclos pedagógicos complementarios (programa general) \\
\hline & Institución Educativa Normal Superior & Ciclos pedagógicos complementarios (programa general) \\
\hline \multirow{4}{*}{ Córdoba } & Universidad de Córdoba & $\begin{array}{c}\text { Licenciatura en Educación Básica con Énfasis en } \\
\text { Humanidades - Lengua Castellana }\end{array}$ \\
\hline & Escuela Normal Santa Teresita & Ciclos pedagógicos complementarios (programa general) \\
\hline & Escuela Normal Superior Lacides Iriarte & Ciclos pedagógicos complementarios (programa general) \\
\hline & Institución Educativa Normal Superior & Ciclos pedagógicos complementarios (programa general) \\
\hline \multirow{2}{*}{ Guajira } & Institución Normal Superior & Ciclos pedagógicos complementarios (programa general) \\
\hline & Institución Educativa Normal Superior Indígena & Ciclos pedagógicos complementarios (programa general) \\
\hline \multirow[t]{3}{*}{ Magdalena } & Universidad del Magdalena & $\begin{array}{l}\text { Licenciatura en Educación Básica con Énfasis en } \\
\text { Humanidades- Lengua Castellana } \\
\text { Licenciatura en Educación Básica con Énfasis en } \\
\text { Matemáticas }\end{array}$ \\
\hline & Institución Educativa Normal Superior San Pedro Alejandrino & Ciclos pedagógicos complementarios (programa general) \\
\hline & Institución Educativa Escuela Normal Superior María Auxiliadora & Ciclos pedagógicos complementarios (programa general) \\
\hline \multirow{5}{*}{ Sucre } & Corporación Universitaria del Caribe. & $\begin{array}{l}\text { Licenciatura en Educación Básica con Énfasis en } \\
\text { Humanidades, Lengua Castellana e Inglés }\end{array}$ \\
\hline & Universidad de Sucre & Licenciatura en Matemáticas \\
\hline & Institución Educativa Normal Superior de Coroza & Ciclos pedagógicos complementarios (programa general) \\
\hline & Institución Educativa Normal Superior de La Mojana & Ciclos pedagógicos complementarios (programa general) \\
\hline & Institución Educativa Normal Superior de Sincelejo & Ciclos pedagógicos complementarios (programa general) \\
\hline
\end{tabular}

Fuente: Elaborado por los autores. 
La muestra de estudio estuvo compuesta por 805 estudiantes encuestados, con un nivel de confianza del 95\% y un margen de error de $\pm 3,5$. Esta muestra fue recolectada a partir de un diseño muestral aleatorio estratificado por departamento, institución y/o programa de formación previamente identificado, a nivel de la región Caribe colombiana, con afijación proporcional, de acuerdo con los siguientes criterios: 1) estudiantes matriculados en programas vinculados con el área pedagógica, y 2) estudiantes del último grado de media vocacional y el ciclo complementario de formación docente en Escuelas Normales Superiores, donde se imparten ciclos pedagógicos complementarios (programas generales). El conjunto de los datos recabados fue procesado y analizado, por medio del programa SPSS.
La tabla 3, nos muestra los resultados obtenidos, a partir de los estadísticos de fiabilidad (alfa de Cronbach) relacionados con las variables generales tenidas en consideración para la medición de los diferentes factores tenidos en cuenta en este trabajo (motivacionales y percepciones), los cuales ponen de manifiesto la solidez del constructo, tenido en cuenta al momento del diseño y ajuste final del instrumento puesto en práctica para la recolección de los datos mostrados en este artículo, al obtener valores superiores a 0,600, salvo en el caso de la variable exigencia de la profesión, la cual fue mantenida, en vista de nuestro interés de establecer comparaciones con resultados obtenidos en otros contextos internacionales, con base a los autores mencionados al comienzo de este apartado.

Tabla 3 - Estadísticos de fiabilidad de los factores de percepción y motivacionales

\begin{tabular}{|c|c|}
\hline Variables incluidas para la medición de los factores de motivación & Alfa de Cronbach \\
\hline Seguridad del trabajo & ,754 \\
\hline Conciliación trabajo-familia & ,794 \\
\hline Movilidad del trabajo &, 558 \\
\hline Valor intrínseco de la carrera & ,717 \\
\hline Influir en el futuro de los niños/adolescentes &, 719 \\
\hline Trabajar en pro de la igualdad social & ,756 \\
\hline Contribución social & ,643 \\
\hline Trabajar con niños/adolescentes &, 814 \\
\hline Habilidad Percibida & ,776 \\
\hline Carrera de segunda opción & 681 \\
\hline Experiencias previas de enseñanza/aprendizaje & ,709 \\
\hline Influencias de conocidos & 810 \\
\hline Variables incluidas para la medición de los factores de percepción & Alfa de Cronbach \\
\hline Carrera exigente & ,696 \\
\hline Profesión exigente &, 566 \\
\hline Estatus social & 841 \\
\hline Salario & ,738 \\
\hline Disuasión social & 688 \\
\hline Satisfacción de la elección & ,826 \\
\hline
\end{tabular}




\section{Resultados}

En primer lugar, daremos cuenta de una exposición descriptiva de la población representada en la muestra para la recopilación de los datos tenidos en cuenta para el abordaje del tema propuesto.

Con el fin de establecer alguno de los rasgos socioeducativos, vinculados a la muestra de estudio diremos que:

- La media de edad $(\mu)$ es equivalente a los 21 años de edad.

- Estuvo conformada mayoritariamente por mujeres (69,3\% versus el 30,7\% de hombres encuestados).

- La mayoría de los encuestados se encontraban estudiando en Escuelas Normales de Superiores (54,8\%), mientras que el 45,2\% estaba en programas de Pedagogía ofertados en universidades.

- El 90,9\% de los encuestados estaban estudiando en programas de Pedagogía impartidos en instituciones educativas oficiales o públicas, mientras que apenas el $9,1 \%$ se encontraba matriculado en instituciones educativas privadas.

- La mayoría $(61,1 \%)$ poseía algún familiar con experiencia docente.

- La mayoría se ubicaba en estratos socioeconómicos muy bajos (el 53,7\% de los encuestados manifestaron residir en zonas de estrato 1), bajo (29,7\% en zonas de estrato 2) o medio bajo (12,2\% en estrato 3); mientras que apenas el 4,3\% se indicaron vivir en estratos socioeconómicos medio alto (estrato 4) o alto (estrato 5).

- 79,8\% de los estudiantes encuestados se encontraban registrados en el SISBEN ${ }^{3}$.

- En cuanto el nivel educativo de los acudientes o representantes legales de los estudiantes encuestados, observamos como el mayor porcentaje de madres $(70,6 \%)$ poseían

3- El SISBEN es el sistema de información establecido por el Gobierno Nacional de Colombia, con el fin de identificar las familias que pueden ser potenciales beneficiarias de programas sociales, con base a su condición socioeconómica estudios primarios $(34,2 \%)$ o bachillerato $(36,4 \%)$, mientras que apenas el $18,6 \%$ contaban con estudios técnicos o tecnológicos o superiores $(10,8 \%$ ), a nivel universitario o posgrado. En cuanto a los padres, el escenario antes descrito se mantiene a grandes rasgos, es decir, la mayoría (68\%) contaban con estudios primarios $(28,7 \%)$ o bachillerato $(39,3 \%)$, mientras que solo el $16,1 \%$ contaba con estudios a nivel técnico y tecnológico y el restante $15,9 \%$ poseían niveles educativos superiores (universitario o posgrado).

- En cuanto a la situación laboral de los representantes legales de los estudiantes encuestados, podemos indicar que los mayores porcentajes de paro o desempleo, se ubican a nivel de las madres de estos (38,6\%), en comparación a los padres (9,3\%); mientras que en ambos casos (tanto en madres como en padres) se observa como el mayor porcentaje de estos trabajan de forma autónoma o independiente $(39,3 \%$ en el caso de las madres y 57,3\% en el caso de los padres), siendo los padres donde se observa el mayor porcentaje de trabajadores por cuenta ajena o asalariados, así como pensionados (27,7\% y 5,7\%, respectivamente), en contraposición a las madres (20,9\% y 2,7\%, respectivamente).

Lo descrito hasta ahora nos permite establecer un perfil básico de la población estudiada, cuyas condiciones socioeducativas nos muestran un contexto caracterizado por estudiantes, predominantemente mujeres, que cuentan con familiares vinculados al sector educativo donde aspiran trabajar luego de finalizar su formación, que se están formando en programas impartidos en instituciones públicas y que presentan condiciones socioeconómicas bajas, marcada por la inestabilidad laboral y un bajo nivel educativo familiar.

La tabla 4 nos muestra las diferentes medias de los puntajes obtenidos en cada uno de los factores puestos a consideración en el marco de este trabajo, al momento de elegir carreras afines al campo pedagógico en Colombia. 
Tabla 4 - Medias de puntajes observados en factores motivacionales y de percepción de los estudiantes encuestados

\begin{tabular}{|c|c|c|}
\hline Factores motivacionales & Media* & Desviación estándar \\
\hline Seguridad del trabajo & 5,24 & 1,33 \\
\hline Conciliación trabajo-familia & 4,27 & 1,39 \\
\hline Movilidad del trabajo & 4,92 & 1,32 \\
\hline Valor intrínseco de la carrera & 5,82 & 1,11 \\
\hline Influir en el futuro de los niños/adolescentes & 6,15 & 0,94 \\
\hline Trabajar en pro de la igualdad social & 5,68 & 1,17 \\
\hline Contribución social & 6,05 & 0,97 \\
\hline Trabajar con niños/adolescentes & 5,69 & 1,29 \\
\hline Habilidad Percibida & 5,86 & 1,08 \\
\hline Carrera de segunda opción & 3,14 & 1,72 \\
\hline Experiencias previas de enseñanza/aprendizaje & 6,04 & 1,03 \\
\hline Influencias de conocidos & 4,57 & 1,76 \\
\hline Factores de percepción & Media* & Desviación estándar \\
\hline Carrera exigente & 6,00 & 1,01 \\
\hline Exigencia de la profesión & 5,65 & 1,15 \\
\hline Estatus social & 4,3 & 1,5 \\
\hline Salario & 2,89 & 1,7 \\
\hline Disuasión social & 4,37 & 1,7 \\
\hline Satisfacción de la elección & 5,92 & 1,18 \\
\hline
\end{tabular}

Fuente: Elaborado por los autores.

Nota: ${ }^{*}$ = La escala tomada para el cálculo de la media obtenida en cada uno de los factores tomados a consideración fue del 1 al 7 , siendo 1 Nada de acuerdo y 7 Muy de acuerdo con cada las consideraciones puestas a valoración por la muestra relacionada con la población de estudio.

Con respecto a los resultados obtenidos para seguida de un valor muy similar por la motivación los tres tipos de motivaciones, el valor que obtiene intrínseca $(5,82)$ y algo por detrás queda la mayor puntuación es la motivación altruista $(5,89), \quad$ motivación extrínseca $(4,81)$ (tabla 5).

Tabla 5 - Medias de puntajes observados en los tres tipos de motivaciones

\begin{tabular}{ccc}
\hline Tipo de motivación & Media & Desviación estándar \\
\hline Motivación extrínseca (valor utilidad personal) & 4,81 & 1,13 \\
Motivación intrínseca (valor intrínseco de la carrera) & 5,82 & 1,11 \\
Motivación altruista (valor de utilidad social) & 5,89 & 0,88 \\
\hline
\end{tabular}

Fuente: Elaborado por los autores. 
Al revisar los factores motivacionales que presentaron mayor valoración entre los estudiantes encuestados, destacan en primer lugar el influir en el futuro de los niños/ adolescentes, contribución social en segundo lugar y experiencias previas de enseñanza/ aprendizaje en tercer lugar. Los factores relacionados con la motivación extrínseca quedan entre los cinco últimos factores junto con los de influencias conocidos y carrera de segunda opción. Cabe destacar que el factor menos valorado ha sido este último.

En cuanto a los factores relacionados con las percepciones que poseían los estudiantes encuestados en torno a la carrera docente, los datos expuestos en la tabla 4, nos permiten ver cómo la visión en torno a la exigencia, al momento de estudiar y ejercer esta profesión, y la satisfacción en la elección de ésta, terminaron siendo muy similares y los que obtienen mayor puntuación; mientras que aquellos factores relacionados con el salario, estatus social obtenido y la disuasión a partir del ejercicio de la labor docente eran los que peores valoraciones presentaron.

\section{Conclusiones}

Como bien exponen autores como Delors (1996), De Moura y Ioschpe (2007), priorizar la calidad de la educación en la mejora de las condiciones sociales a nivel mundial, en nuestro caso en Colombia, requiere reconocer el papel que cumplen los docentes en la mejora del sector educativo requerido para tal fin. Para ello, como expusimos al principio de este trabajo, la identificación de los factores que inciden en la elección carreras pedagógicas en el país estudiado es de vital importancia para diseñar e implementar estrategias orientadas a atraer a los mejores estudiantes para el ejercicio de esta función, en los términos expuestos por Barber y Mourshed (2007). Ello, como bien destacan Sahlberg (2010) y Beca y Cerri (2014), por una parte, bajo una agenda política educativa marcada por la falta de relevancia de los docentes, al momento de diseñar las políticas educativas a nivel regional y a nivel colombiano, en nuestro caso; y por otra bajo la necesaria promoción de un modelo de formación que propenda a la mayor calidad de los futuros docentes, bajo un contexto social que garantice un aumento de la dignidad profesional y respeto social.

Los resultados obtenidos, a nivel del perfil de los futuros docentes analizados en Colombia, no solo reafirmarían parte de lo destacado por autores como Tenti (2007) o Bellei y Valenzuela (2010), sino que ahondarían la auto-percepción social que presentan los miembros de la población aquí estudiada, al ubicarse en contextos sociales de nivel bajo, caracterizados por un núcleo familiar inestable económicamente y de bajo nivel formativo. Algo que traería consigo un marco de transformación educativa que difícilmente redundaría en una mayor calidad educativa, hasta tanto no se garantice de mecanismos que incentiven el ingreso de estudiantes con condiciones socioeducativas más favorables para acometer un cambio significativo y positivo al sector educativo donde se insertarán, una vez concluida su formación como docentes. Lo aquí expuesto se hace reiterando que, si bien el Gobierno Nacional de Colombia, a través del Ministerio de Educación, ha impulsado en los últimos años programas a favor de la formación de futuros docentes en el área de educación infantil ${ }^{4}$ y a nivel de posgrado ${ }^{5}$, el impacto que pueden traer este tipo de medidas seguirá siendo limitado. Esto ocurre en vista de la falta de su continuidad en el tiempo y por su carácter restringido a un número limitado de estudiantes y programas académicos. Esto hace que el problema de fondo, la formación de futuros docentes poco calificado siga generándose en dicho país. $\mathrm{Al}$ menos hasta que no se avance, como bien

4- En 2012 el Ministerio de Educación Nacional de Colombia promovió becas para la formación de 6.000 estudiantes de bachillerato interesados en estudiar carrera docente a nivel de educación infantil.

5- En 2015, el Ministerio de Educación Nacional de Colombia abrió convocatoria de 4.500 créditos becas para la formación de docentes a nivel de maestría en universidades colombianas, a partir de 2016 hasta 2018. 
apuntan Gatti, Siqueira y Dalmazo (2011), en la promoción de una política docente al interior de la agenda política educativa dispuesta a nivel nacional, que contribuya no solo a la mejora de las condiciones salariales e incentivos, sino en la búsqueda de mecanismos que garanticen programas curriculares de calidad que atraigan a estudiantes mejor calificados (CEPAL; UNESCO, 1996; VAILLANT, 2004; OREALC/UNESCO, 2012). Sobre todo si lo que se quiere es favorecer un marco de constitución de futuros docentes, caracterizado por altos niveles de abandono y deserción durante su proceso de formación (ICFES, 2011; COLOMBIA, 2016).

Otro aspecto que nos ayuda a ahondar en el análisis de los resultados expuestos en este trabajo, son los factores que motivan la elección de la carrera docente. En primer lugar, los datos reafirmarían lo expuesto por Calvo (2006) y Vaillant (2013), en lo que se refiere a la elección por descarte de programas de formación en el campo educativo. El hecho que una mayoría de encuestados se sitúe en un estrato socioeconómico muy bajo y que más de un 70\% esté registrado en el SISBEN, aunado con la escasa formación del entorno familiar, nos puede dar una idea de la forma como estaría siendo concebida carreras afines al sector educativo: como programas formativos que estarían siendo accedidos como un ascensor social o mejora de las bajas condiciones socioeconómicas que ya tienen los futuros docentes, desde la estabilidad laboral que pudiesen brindar este tipo de carreras.

Por último, los datos nos permiten confirmar hipótesis, no solamente expuestas a nivel colombiano (GARCÍA et al., 2014), nuestro caso de estudio; sino también por autores como Avendaño y González (2012), Ryan y Deci (2000) y Gratacós (2014) tanto a nivel latinoamericano como a nivel internacional, en lo que se refiere al tipo de motivaciones que son tenidas en cuenta al momento de escoger carreras afines al campo educativo, al observarse cómo los factores motivacionales predominantes en los futuros docentes se enmarcan en argumentos de tipo intrínsecos y altruistas, más que de carácter personal, al menos en el caso de los estudiantes encuestados. Este hecho nos marca un contexto donde la promoción de políticas docentes, como las indicadas al comienzo de este apartado, deberían ir aparejadas con una agenda política nacional que promueva un contexto social que centre su mirada también en la búsqueda de mecanismos que ayuden a romper la paradoja social existente en la actualidad, donde, pese al importante rol que tienen los docentes en la mejora de los resultados de aprendizaje demostrados por los estudiantes, esto no se estaría correspondiendo a ser reconocido socialmente o al menos a como se auto-perciben quienes se están formando para ejercer como tales. Algo que estaría afectando profundamente en la atracción de los estudiantes con mayores talentos en el ejercicio de la carrera docente; además de incidir en la promoción de contextos de disuasión social, que estaría contribuyendo al abandono de estudiantes, durante el período formativo, y en los primeros años de la profesión (ÁVALOS et al., 2010; UNESCO, 2012). Todo ello a pesar de la estabilidad laboral que pudiese brindar este tipo de profesiones (altamente valoradas por los estudiantes encuestados), en los términos expuestos por Falus y Goldberg (2011), y del impacto potencial que estarían trayendo consigo la influencia que tendrían agentes educativos que han marcado favorablemente el proceso de selección de quienes deciden tomar el camino de la enseñanza.

\section{Referencias}

ÁVALOS, Beatrice. La formación inicial docente en Chile: tensiones entre políticas de apoyo y control. Estudios Pedagógicos, Valdivia, n. 40, n. especial, p. 11-28, 2014.

ÁVALOS, Beatrice et al. La profesión docente: temas y discusiones en la literatura internacional. Estudios Pedagógicos, Valdivia, n. 36, p. 235-263, 2010. 
AVENDAÑO, Cecilia; GONZÁLEZ, Rodrigo. Motivos para ingresar a las carreras de Pedagogía de los estudiantes de primer año de la Universidad de Concepción. Estudios Pedagógicos, Valdivia, n. 38, n. 2, p. 21-33, 2012.

BARBER, Michael; MOURSHED, Mona. How the world's best performing schools systems come out on top. London: McKinsey, 2007.

BARÓN, Juan; BONILLA, Leonardo. Profesionalización docente y calidad de la educación escolar en Colombia. Bogotá: Banco de la República, 2011. (Documentos de trabajo sobre economía regional, 154). Disponible en: <http://www.banrep.gov.co/ documentos/publicaciones/regional/documentos/DTSER-154.pdf>. Acceso: 20 ago, 2016.

BARRERA-OSORIO, Felipe; MALDONADO, Darío; RODRIGUEZ, Catherine. Calidad de la educación básica y media en Colombia: diagnóstico y propuesta. Bogotá: Universidad de los Andes, 2012. (Documentos CEDE, 41). Disponible en: <http://cippec.org/ mapeal/wp-content/uploads/2014/05/BARRERA-OSORIO-y-otros-Calidad-de-la-educ-en-Colombia.pdf>. Acceso en: 15 feb. 2016.

BECA, Carlos; CERRI, Marianela. Políticas docentes como desafío de educación para todos más allá del 2015: apuntes. Santiago de Chile: [s. n.], 2014. (Educación y desarrollo post-2015, 1). Disponible en: <http://www.unesco.org/new/fileadmin/ MULTIMEDIA/FIELD/Santiago/pdf/Apuntes-2014-n1-carlos-eugenio-beca-esp.pdf.>. Acceso en: 5 en. 2016.

BELLEI, Cristián; VALENZUELA, Juan Pablo. ¿Están las condiciones para que la docencia sea una profesión de alto estatus en Chile? In: MARTINIC, Sergio; ELACQUA, Gregory (Ed.). ¿Fin de ciclo? Cambios en la gobernanza del sistema educativo. Santiago de Chile: OREAL-UNESCO, 2010. p. 257-284.

BERGER, Jean-Louis; D'ASCOLI, Yannick. Motivations to become vocational education and training educators: a person-orientated approach. Vocations and Learning, Reino Unido, v. 5, n. 3, p. 225-249, 2012.

BROOKHART, Susan; FREEMAN, Donald. Characteristics of entering teaching candidates. Review of Educational Research, Washington, n. 62, n. 1, p. 37-60, 1992.

CALVO, Gloria. La profesionalización docente en Colombia. Bogotá: Fundación Compartir, 2006.

CARLO, Alain et al. Study on policy measures to improve the attractiveness of the teaching profession in Europe. Bruselas: Comisión Europea, 2013.

CEPAL; UNESCO. Educación y conocimiento: eje de la transformación productiva con equidad. Santiago de Chile: Autores, 1996.

COLOMBIA. Ministerio de Educación Nacional de Colombia - MENC. Sistema para la prevención de la deserción de la educación superior. Bogotá: Autor, 2016. Disponible en: <http://spadies.mineducacion.gov.co/spadies/>. Acceso en: 5 sept. 2016.

COX, Cristián; BECA, Carlos; CERRI, Marianela. Docentes para una educación de calidad en América Latina: Problemas y orientaciones de política. In: DÍAZ DE LA TORRE, Juan (Comp.). Realidades y prospectiva educativa. Tomo I. México: SNTE, 2014. p. 41-92.

DELORS, Jaques. La educación encierra un tesoro. Madrid: Santillana-Unesco, 1996.

DE MOURA, Claudio; IOSCHPE, Gustavo. La remuneración de los maestros en America latina: ¿es baja?, ¿afecta la calidad de la enseñanza? Santiago de Chile: PREAL, 2007.

DESHANO, Carol. Reclutamiento de docentes: orientaciones para el diseño de las políticas en América Latina. Santiago de Chile: PREAL, 2012.

ECCLES, Jacquelynne et al. Expectancies, values, and academic behaviors, In: SPENCE, Janet (Ed.). Achievement and achievement motives: psychological and sociological approaches. San Francisco: Freeman, 1993. p. 75-146.

EREN, Altay; TEZEL, Kadir. Factors influencing teaching choice, professional plans about teaching, and future time perspective: a meditational analysis. Teaching and Teacher Education, v. 26, n. 7, p. 1416-1428, oct. 2010.

FALUS, Lucila; GOLDBERG, Mariela. Perfil de los docentes en América Latina. Buenos Aires: SITEAL, 2011. 
FELDFEBER, Myriam. La regulación de la formación y el trabajo docente: un análisis crítico de la "agenda educativa" en América Latina. Educação \& Sociedade, Campinas, v. 28, n. 99, p. 444-465, 2007.

FOKKENS-BRUINSMA, Marjon; CANRINUS, Esther. Adaptative and maladaptative motives for becoming a teacher. Journal of Education for Teaching, n. 38, n. 1, p. 3-19, feb. 2012.

GARCÍA, Sandra et al. Tras la excelencia docente: cómo mejorar la calidad de la educación para todos los colombianos. Bogotá: Fundación Compartir, 2014.

GATTI, Bernardete et al. Formação de professores para 0 ensino fundamental: instituições formadoras e seus currículos. São Paulo: Fundação Carlos Chagas: Fundação Vitor Civita, 2008. Relatório de pesquisa.

GATTI, Bernardete; SIQUEIRA, Elba; DALMAZO, Marli. Políticas docentes no Brasil. Brasília, Unesco, 2011.

GRATACÓS, Gloria. Estudio sobre las motivaciones en la elección de ser maestro, 2014. 357 p. Tesis (Doctorado) - Universidad Internacional de Cataluña, Barcelona, 2014.

GRATACÓS, Gloria; LÓPEZ-JURADO, Marta. Validación de la versión en español de la escala de los factores que influyen en la elección de los estudios de educación (FIT-choice). Revista de Educación, Madrid, n. 372, p. 87-105, 2016.

HANUSHEK, Erik. The economic value of higher teacher quality. Economics of Education Review, v. 30, n. 3, p. 466-479, 2011.

HUNT, Barbara. Efectividad del desempeño docente: una reseña de la literatura internacional y su relevancia para mejorar la educación en América Latina. Santiago de Chile: PREAL, 2009.

ICFES - Instituto Colombiano para la Evaluación de la Educación. Exámenes de estado de calidad de la educación superior SABER PRO: resultados del periodo 2005-2009. Bogotá: Icfes, 2011. Disponible en: <http://www.icfes.gov.co/index.php/ investigadores-posgrado/informes-de-resultados-evaluaciones-nacionales/saber-pro.>. Acceso en: 2 sept. 2016.

JUGOVIĆ, Ivana et al. Motivation and personality of preservice teachers in Croatia. Asia-Pacific Journal of Teacher Education, v. 40 , n. 3, p. 271-287, 2013.

KLASSEN, Robert et al. Investigating pre-service teacher motivation across cultures using the teachers' ten statements test. Teaching and Teacher Education, v. 27, n. 3, p. 579-588, apr. 2011.

KÖNIG, Johannes; ROTHLAND, Martin. Motivations for choosing teaching as a career: effects on general pedagogical knowledge during initial teacher education. Asia-Pacific Journal of Teacher Education, v. 40, n. 3, p. 289-316, 2012.

KRISHNARATNE, Shari; WHITE, Howard; CARPENTER, Ella. Quality education for all children? What works in education in developing countries. [S. I.]: International Initiative for Impact Evaluation, 2013.

LIANG, Xiaoyan. Teacher pay in 12 Latin American Countries: how does teacher pay compare to other professions, what determines teacher pay, and who are the teachers? Washington, DC.: World Bank, 1999.

LIN, Emily et al. Initial motivations for teaching: comparison between preservice teachers in the Unites States and China. AsiaPacific Journal of Teacher Education, v. 40, n. 3, p. 227-248, 2012.

LÓPEZ-JURADO, Marta; GRATACÓS, Gloria. Elegir enseñar: propuesta del modelo antropológico de la motivación de Pérez López aplicada al ámbito de la educación. Estudios sobre Educación, Navarra, v. 24, p. 125-147, 2013.

NUMA, Sergio. Salarios de maestros, ¿Quién tiene la razón? Bogotá: El Espectador, 2015. Disponible en: <http://www. elespectador.com/noticias/educacion/salarios-de-maestros-quien-tiene-razon-articulo-557448>. Acceso en: 25 en. 2016.

OREALC/UNESCO. Antecedentes y criterios para la elaboración de políticas docentes en América Latina y el Caribe: proyecto estratégico regional sobre políticas docentes. Santiago de Chile: OREALC/UNESCO, 2012.

RIVKIN, Steven; HANUSHEK, Eric; KAIN, John. Teachers, schools, and academic achievement. Econometría, v. 73, n. 2, p. 417-458, 2005. 
RYAN Richard; DECl, Edward. Self-determination theory and the facilitation of intrinsic motivation, social development, and wellbeing. American Psychologist, v. 55, n. 1, p. 68-78, jan. 2000.

SAHLBERG, Pasi. The secret to finland's success: educating teachers. Stanford: Stanford Center for Opportunity Policy in Education, 2010.

SCHMELKES, Sylvia. La evaluación del desempeño docente. In: CONGRESO INTERNACIONAL, 2. 2014, Guadalajar. Guadalajara. Actas do congreso internacional "Competencias para la innovación educativa". Guadalajara: Unives, 2014. Disponible en: $<$ http://unives.com.mx/conferencia-magistral-por-la-dra-sylvia-schmelkes-la-evaluacion-del-desempeno-docente>. Acceso en: 20 feb. 2016.

SCHWARTZMAN, Simon. La institucionalización de las políticas docentes en América Latina. Rio de Janeiro: Instituto de Estudos do Trabalho e Sociedade, 2011. Disponible en: <https://archive.org/details/ LalnstitucionalizacinDeLasPolticasDocentesEnAmricaLatina>. Acceso en: 12 dic. 2015.

TENTI, Emilio. La condición docente: análisis comparado de la Argentina, Brasil, Perú y Uruguay. Buenos Aires: Siglo XXI, 2007.

TOPKAYA, Ece; UZTOSUN, Mehmet. Choosing teaching as a career: motivations of pre-service English teachers in Turkey. Journal of Language Teaching and Research, v. 3, n. 1, p. 126-134, jan. 2012.

UNESCO. Antecedentes y criterios para la elaboración de políticas docentes en América Latina y el Caribe. París: Unesco, 2012.

UNESCO. La educación para todos, 2000-2015: logros y desafíos. París: Unesco, 2015.

VAILLANT, Denise. Construcción de la profesión docente en América Latina: tendencias, temas y debates. Santiago de Chile: Preal, 2004.

VAILLANT, Denise. Formación inicial del profesorado en América Latina: dilemas centrales y perspectivas. Revista Española de Educación Comparada, Madrid, n. 22, p. 185-206, 2013.

VAILLANT, Denise. Políticas docentes en América Latina: elementos para el debate. In: MARTínEZ LARRECHEA, Enrique; CHIANCONE, Adriana. Políticas educativas en el cono sur. Montevideo: Magro, 2010. p. 149-176.

VAILLANT, Denise; MARCELO, Carlos. Ensinando a ensinar: as quarto etapas de uma aprendizagem. Curitiba: UFPR, 2012.

VALLE, Javier. La Unión Europea y su política educativa. Madrid: Ministerio de Educación y Ciencia, 2006.

VARKEY GEMS FOUNDATION. 2013 global teacher status index. London: Varkey GEMS Foundation, 2013.

WATT, Helen; RICHARDSON, Paul. Motivational factors influencing teaching as a career choice: development and validation of the FIT-choice scale. The Journal of Experimental Education, v. 75, n. 3, p. 167-202, 2007.

WATT, Helen et al. Motivations for choosing teaching as a career: an international comparison using the FIT-choice scale. Teaching and Teacher Education, v. 28, n. 6, p. 791-805, aug. 2012.

Recibido en: 10.03 .2016

Aprobado en: 13.09.2016

Elias Said-Hung es doctor en ciencias de la información con más de 10 años de experiencia profesional en los medios de comunicación social, medios digitales y las TIC en la educación. Es profesor titular de la Facultad de Educación de la Universidad Internacional de la Rioja.

Gloria Gratacós es doctora en educación, licenciada en económicas por la Universidad de Barcelona, licenciada en ciencias empresariales y máster en dirección de empresas por ESADE. Es directora del área de Educación y Psicología del C.U. Villanueva (adscrito a a la Universidad Complutense de Madrid).

Jorge Valencia Cobos es máster en educación con énfasis en medios y educación, economista en su formación básica. Actualmente es coordinador de investigación del Observatorio de Educación de la Universidad del Norte. 